\title{
Enquête sur les sérotypes de Pasteurella multocida dans les zones de savane du Cameroun
}

\author{
N.L. Njongmeta ${ }^{1}$ V.N. Tanya ${ }^{1 *}$ A. Keng ${ }^{1}$
}

Mots-clés

Bovin - Pasteurella multocida Sérotype - Septicémie hémorragique Cameroun.

\begin{abstract}
Résumé
Une étude a été menée sur l'occurrence des sérotypes de Pasteurella multocida dans trois grandes zones d'élevage bovin du Cameroun. Au total, 362 échantillons de sérum bovin ont été collectés dans les provinces de I'Extrême-Nord, de l'Adamaoua et du Nord-Ouest, et testés afin de détecter la présence d'anticorps vis-à-vis de cinq sérotypes de P. multocida (A5, A7, B1, B6 et E6) par le test d'hémagglutination. Le sérotype B6 a été présent dans toutes les régions, avec la fréquence la plus élevée $(84,4$ p. 100) dans la région de Mora et la plus faible (43,6 p. 100) dans celle de Meiganga. Les autres sérotypes ont aussi été présents mais à des niveaux différents. Les résultats montrent la nécessité d'incorporer le sérotype B6 dans le vaccin contre la septicémie hémorragique actuellement utilisé au Cameroun.
\end{abstract}

\section{INTRODUCTION}

La septicémie hémorragique est une forme aiguë, septicémique et très souvent fatale de la pasteurellose affectant essentiellement les buffles d'eau et les bovins en Asie et en Afrique. Elle se manifeste par des épidémies, particulièrement au cours de périodes de stress environnemental et d'épuisement. Elle est économiquement importante, surtout dans les zones où la maladie est endémique, et se caractérise cliniquement par une poussée soudaine de fièvre, une salivation profuse, une sévère dépression et la mort dans les

1. Laboratoire associé francophone (Laf 309), Centre régional de recherche
agricole pour le développement de Wakwa, BP 65, Ngaoundéré, Cameroun

* Adresse actuelle de l'auteur pour la correspondance

Centre de recherche agricole pour le développement de Bambui, BP 51, Bamenda, Cameroun

Tél. : (237) 7769083 ; e-mail : vntanya@yahoo.com
24 heures. Les taux de morbidité et de mortalité varient entre 50 et 100 p. 100 dans un troupeau cliniquement infecté. Les animaux guéris passent par une longue convalescence et peuvent devenir des porteurs sains, mais aussi parfois servir de foyers pour de nouvelles apparitions épidémiques (5).

La maladie est causée par Pasteurella multocida. Plusieurs sérotypes existent dont les cinq principaux, désignés A, B, C, D et E, ont été identifiés grâce à un test d'hémagglutination indirecte $(1,2)$. Le sérotype B6 cause la septicémie hémorragique en Asie et en Afrique (Egypte, Ethiopie, Soudan), tandis que le sérotype E6 est la cause de la maladie au Cameroun et dans le reste de l'Afrique (1, 5). En 1992, Martrenchar et Njanpop (6) ont isolé le sérotype B6 dans le nord du Cameroun. C'était la première isolation de ce sérotype à l'ouest du Soudan.

Cette étude a eu pour objectif d'évaluer l'étendue de l'incursion du sérotype B6 au Cameroun et l'occurrence des autres sérotypes afin de suggérer des mesures de contrôle appropriées. 


\section{MATERIEL ET METHODES}

\section{Collecte des échantillons de sérum}

Les échantillons ont été collectés à Yagoua et Mora dans la province de l'Extrême-Nord, Meiganga et Banyo dans la province de l'Adamaoua, et Sabga (près de Bamenda) dans la province du Nord-Ouest. Le sang de bovins adultes a été collecté par ponction au niveau de la jugulaire dans des tubes vacutainer et le sérum stocké dans des fioles à $-20^{\circ} \mathrm{C}$. Alors que les échantillons de sang de Yagoua, Mora et Banyo avaient été prélevés dans des troupeaux sédentaires, ceux de Meiganga et Sabga provenaient du marché à bétail.

\section{Préparation des érythrocytes}

Les hématies humaines de type $\mathrm{O}$, qui sont plus appropriées pour utilisation dans ce test, ont été obtenues à partir du sang périmé provenant de la banque de sang de l'Hôpital protestant de Ngaoundéré. Les hématies de plusieurs espèces animales contiennent l'antigène hétérophile qui est aussi présent dans $P$. multocida. Ces hématies ont été lavées trois fois dans six volumes de solution saline. Les cellules tassées issues du dernier lavage ont été conservées au réfrigérateur et utilisées selon les besoins pendant une période de trois jours (1).

\section{Préparation de l'extrait bactérien}

Des souches de cultures de divers sérotypes de P. multocida ont été obtenues auprès du Laboratoire national vétérinaire (Lanavet) de Boklé-Garoua (Cameroun) et cultivées sur gélose au sang (7 p. 100 de sang de cheval) afin d'obtenir une prolifération confluente à 18-24 h. La culture a été récoltée après grattage de la gélose et déposée dans $4 \mathrm{ml}$ d'une solution saline normale, chauffée à $56^{\circ} \mathrm{C}$ pendant 30 min afin de dissoudre les antigènes de surface, puis centrifugée, et le surnageant enlevé et conservé dans un autre tube (1).

\section{Sensibilisation des hématies}

A l'extrait bactérien (surnageant) d'un volume d'environ $3 \mathrm{ml} \mathrm{a}$ été ajouté $0,2 \mathrm{ml}$ des érythrocytes lavés et tassés. Cellules et extrait ont été soigneusement mélangés et placés dans un incubateur à $37{ }^{\circ} \mathrm{C}$ pendant $2 \mathrm{~h}$. Les hématies ont ensuite été séparées par centrifugation et lavées avec $10 \mathrm{ml}$ de solution saline normale. Une quantité suffisante de solution saline a été ajoutée aux érythrocytes sensibilisés afin de produire une suspension de 1 p. 100 (1).

\section{Test d'hémagglutination}

Le sérum a été testé pour la présence d'anticorps vis-à-vis de divers sérotypes de $P$. multocida grâce au test d'hémagglutination indirecte. Le test a été réalisé dans des plaques de microtitration comme décrit par Carter (1). Des volumes égaux de dilution sérique $(1: 10,1: 20,1: 40,1: 80$ et 1:100) et 1 p. 100 d'hématies humaines de type $\mathrm{O}$ ont été ajoutées dans chaque cupule, tandis que des volumes équivalents de la suspension d'hématies humaines de type $\mathrm{O}$ ont été ajoutés dans des cupules contenant seulement du Pbs et devant servir de témoin. Les plaques de microtitration ont été secouées puis laissées à la température ambiante pendant $2 \mathrm{~h}$ avant la lecture des premiers résultats. L'hémagglutination a indiqué un résultat positif. Les plaques ont ensuite été placées dans le réfrigérateur pour la nuit et, le jour suivant, elles ont été doucement secouées, laissées à reposer puis lues en vue de confirmer les résultats du jour précédent. Le titre était la valeur réciproque de la plus grande dilution donnant une hémagglutination de 100 p. 100 de la suspension de 1 p. 100 d'hématies humaines de type $\mathrm{O}$.

\section{RESULTATS ET DISCUSSION}

Au total, 362 échantillons de sérum bovin ont été testés pour la présence d'anticorps vis-à-vis de cinq sérotypes de $P$. multocida. Tous les cinq sérotypes ont montré une réaction d'hémagglutination positive avec un certain nombre de sérums (tableau I).

Le sérotype A5 a été largement prédominant dans la zone de Mora (81,3 p. 100) mais l'a moins été à Meiganga (21,8 p. 100). Le sérotype A7 a eu une prédominance de 40,6 p. 100 à Mora mais a été complètement absent de la région de Yagoua. Le sérotype B1 a été le plus présent dans la région de Sabga avec une prévalence de 57,1 p. 100 , contre 18,9 p. 100 dans la région de Yagoua. Le sérotype E6 a été prédominant dans la région de Yagoua (73,0 p. 100) et moins présent dans la région de Meiganga. Le sérotype B6 quant à lui a été fortement prédominant dans la zone de Mora (84,4 p. 100) et moins présent à Meiganga. Dans l'ensemble, le sérotype B6 a montré une prédominance constante dans toutes les régions. La méthode utilisée dans cette étude a été en accord avec celle de Carter $(1,2)$, où l'hémagglutination est considérée comme positive à un taux de dilution de sérum de 1:100. A ce titre d'agglutination, les agglutinations non spécifiques sont minimisées (4).

Ces résultats ont confirmé que le sérotype B6 de $P$. multocida était présent au Cameroun, comme cela a été rapporté par Martrenchar et Njanpop (6) qui ont signalé pour la première fois la présence de ce sérotype dans la zone de Yagoua dans la province de l'ExtrêmeNord camerounais. Ce sérotype était présent dans les cinq régions concernées par cette étude. Le sérotype E6 est connu pour causer la septicémie hémorragique au Cameroun. Les vaccinations annuelles sont donc destinées à lutter contre E6. De ce fait, il est

\section{Tableau I}

Prévalence de différents sérotypes de Pasteurella multocida dans quelques régions du Cameroun

\begin{tabular}{|c|c|c|c|c|c|}
\hline \multirow[t]{2}{*}{ Région } & \multicolumn{5}{|c|}{ Sérotype } \\
\hline & A5 & A7 & B1 & B6 & E6 \\
\hline Yagoua $(\mathrm{N}=74)$ & $34(45,9 \%)$ & $0(0,0 \%)$ & $14(18,9 \%)$ & $40(54,1 \%)$ & $54(73,0 \%)$ \\
\hline Mora $(N=64)$ & $52(81,3 \%)$ & $26(40,6 \%)$ & $32(50,0 \%)$ & $54(84,4 \%)$ & $40(62,5 \%)$ \\
\hline Meiganga $(N=110)$ & $24(21,8 \%)$ & $2(1,8 \%)$ & $30(27,3 \%)$ & $48(43,6 \%)$ & $44(40,0 \%)$ \\
\hline Banyo ( $N=72)$ & $36(50,0 \%)$ & $14(19,4 \%)$ & $18(25,0 \%)$ & $50(69,4 \%)$ & $38(52,8 \%)$ \\
\hline Sabga $(N=42)$ & $28(66,7 \%)$ & $10(23,8 \%)$ & $24(57,1 \%)$ & $30(71,4 \%)$ & $22(52,4 \%)$ \\
\hline
\end{tabular}


possible que les titres de E6 mesurés dans cette étude soient un reflet de cette vaccination plutôt que d'une prévalence de maladie active. Les autres sérotypes, quoique présents, ne posent pas le même risque sanitaire aux populations bovines que les sérotypes E6 et B6. Cette étude indique la présence d'une grande variété de sérotypes au Cameroun.

Ainsi que l'avaient suggéré Martrenchar et Njanpop (6), le sérotype B6 peut avoir été introduit au Cameroun à partir de l'Afrique de l'Est grâce à des mouvements transfrontaliers incontrôlés des troupeaux transhumants ou commerciaux. Ce même phénomène aurait aidé à sa dispersion à partir du point d'entrée. Les présents résultats indiquent qu'il serait avantageux qu'un vaccin bivalent contenant à la fois B6 et E6 soit développé afin de prévenir de futures épidémies d'hémorragie septicémique au Cameroun.

\section{Remerciements}

Les auteurs sont reconnaissants à l'Agence universitaire de la francophonie (Auf) qui a financé ce travail dans le cadre d'un contrat d'association du Laboratoire associé francophone (Laf 309) et du Fonds francophone de la recherche (Ffr). Ils remercient également le Dr Yaya du Lanavet de Boklé-Garoua qui leur a procuré les souches de culture de divers sérotypes de $P$. multocida.

\section{Summary}

Njongmeta N.L., Tanya V.N., Keng A. Survey on Pasteurella multocida Serotypes in the Savanna Zones of Cameroon

A study was carried out on the occurrence of Pasteurella multocida serotypes in three major cattle breeding zones of Cameroon. A total of 362 cattle serum samples were collected in the Far North, Adamawa and North West provinces. They were tested with the hemagglutination test for the presence of antibodies against five $P$. multocida serotypes (A5, A7, B1, B6 and E6). Serotype B6 was present in all the regions with the highest prevalence $(84.4 \%)$ in the region of Mora and the lowest $(43.6 \%)$ in that of Meiganga. The other serotypes were also present with varying intensities. These results show the need to incorporate serotype B6 in the currently used hemorrhagic septicemia vaccine in Cameroon.

Key words: Cattle - Pasteurella multocida - Serotype Hemorrhagic septicemia - Cameroon.

\section{BIBLIOGRAPHIE}

1. CARTER G.R., 1955. Studies of Pasteurella multocida. 1. Haemagglutination test for the identification of serological types. Am. J. vet. Res., 61: 481-184.

2. CARTER G.R., 1961. A new serological type of Pasteurella multocida from Central Africa. Vet. Rec., 73: 1052.

3. DE ALWIS M.C.L., 1984. Haemorrhagic septicaemia in cattle and buffaloes. Revue sci. tech. Off. int. Epizoot., 3: 707-730.

4. HUSSEIN A.M., ELSAWI M.O., 1984. A serological survey of sheep sera for antibodies to Pasteurella haemolytica serotypes in the Sudan. Revue Elev. Méd. vét. des Pays trop., 37: 418-421.

5. LEFEVRE P.C., 1991. Atlas des maladies infectieuses des ruminants. Maisons-Alfort, France, Cirad-iemvt, p. 56-57.

6. MARTRENCHAR A., NJANPOP B.M., 1994. Première observation d'une épidémie de septicémie hémorragique due à Pasteurella multocida sérotype B6 au Nord Cameroun. Revue Elevage Méd. vét. Pays trop. $47: 19-20$.

Reçu le 18.10.2001, accepté le 11.07.2002

\section{Resumen}

Njongmeta N.L., Tanya V.N., Keng A. Encuesta sobre los serotipos de Pasteurella multocida en las zonas de sabana de Camerún

Se llevó a cabo un estudio sobre la presencia de serotipos de Pasteurella multocida en tres grandes zonas de cría bovina en Camerún. En total, se recolectaron 362 muestras de suero bovino en las provincias del Extremo norte, de Adamaoua y de Noroeste y fueron luego estudiadas con el fin de detectar la presencia de anticuerpos de cinco serotipos de $P$. multocida (A5, A7, B1, B6 y E6) mediante el test de hemoaglutinación. El serotipo B6 estuvo presente en todas las regiones, con la frecuencia más elevada $(84,4 \%)$ en la región de Mora y la más baja $(43,6 \%)$ en la de Meiganga. Los otros serotipos estuvieron también presentes, pero a niveles diferentes. Los resultados muestran la necesidad de incorporar el serotipo B6 en la vacuna contra la septicemia hemorrágica actualmente utilizada en Camerún.

Palabras clave: Ganado bovino - Pasteurella multocida Serotipo - Septicemia hemorrágica - Camerún. 